\title{
Kawasaki Disease Complicated by Peripheral Gangrene in a Case of Inherited Thrombophilia
}

\author{
Faten Al Tasseh ${ }^{\mathrm{a}}$, Hassan El Khatib ${ }^{\mathrm{a}, \mathrm{d}}$, Rodaina Kordab ${ }^{\mathrm{a}}$, Soha Ghanem ${ }^{\mathrm{a}}$, Ziad Naja ${ }^{\mathrm{a}}$, \\ Ahmad Salaheddine Naja ${ }^{b}$, Georges Haber ${ }^{c}$, Mariam Rajab ${ }^{\mathrm{a}}$, \\ Malek Baassiria ${ }^{a}$ Rachid Haidar ${ }^{b}$
}

\begin{abstract}
Kawasaki disease is a febrile vasculitis targeting medium sized arteries, causing coronary artery aneurysm without appropriate treatment. Many children do not fulfill the criteria and due to its serious complications, the American Heart Association (AHA) published an algorithm for atypical Kawasaki disease based on laboratory tests and echocardiography. Peripheral ischemia is a rare complication with harmful sequalae. Here we reports a case of a 14-month-old patient, who was diagnosed of atypical Kawasaki disease, developed peripheral ischemia and gangrene of the second and third right hand fingers, and was found to have factor V Leiden heterozygous mutation.
\end{abstract}

Keywords: Kawasaki; Gangrene; Thrombophilia; Factor V Leiden; Ileal atresia

\section{Introduction}

Kawasaki disease is an acute systemic vasculitis primarily affecting infants and young children. Twenty percent of children with this disease who do not receive intravenous gamma globulin (IVIG) therapy develop coronary artery aneurysms [1]. Peripheral ischemia and necrosis are rare and harmful complications, which are reported mainly below age of 1 year. We describe here a case of a 14-month-old male, who were diagnosed of atypical Kawasaki disease, and developed peripheral ischemia and gangrene of the second and third right hand fingers, with factor V Leiden heterozygous mutation.

Manuscript submitted June 23, 2018, accepted August 1, 2018

aPediatric Department, Makassed General Hospital, Beirut, Lebanon bOrthopaedic Surgery Division, American University of Beirut Medical Center, Beiut, Lebanon

'Orthopaedic Surgery, Mount Lebanon Hospital, Lebanon

${ }^{\mathrm{d} C}$ Corresponding Author: Hassan El Khatib, Pediatric Department, Makassed General Hospital, Beirut, Lebanon. Email: hassan-khatib@hotmail.com

doi: https://doi.org/10.14740/ijcp306w

\section{Case Report}

This is a case of a 14-month-old male patient, with no significant family history. Prenatally, an ultrasound revealed a dilated ascending colon and mild polyhydramnios. He was born at full term 39 weeks, by normal vaginal delivery to gravida 2 , para 2 and aborta 0 , vigorous with Apgar $=9$ and 10 at $1 \mathrm{~min}$ and 5 min respectively and weight at birth was $3.7 \mathrm{~kg}$ (50th percentile). He was found to have distended abdomen on physical exam shortly after birth; kidney, ureter and bladder X-ray (KUB) showed air-fluid level and laparotomy was done at day 2 of life, which revealed ileal atresia and resection of $15 \mathrm{~cm}$ with primary anastomosis was done without complications.

The patient presented, to another institution, with a fever of 5-day duration, where he was diagnosed of otitis media and received three doses of ceftriaxone; fever subsided for 1 day and then redeveloped, so he presented to our care. Upon admission, his weight was $10.2 \mathrm{~kg}(10-25$ th percentile) and his height was $81 \mathrm{~cm}$ (50th percentile). He was still febrile (temperature $39^{\circ} \mathrm{C}$ ) and the rest of vital signs were within normal limits: pulse was 125 beats/min, respiratory rate was 30 cycles/ min and his blood pressure was 90/50 mm Hg (90 - 105/55 $70 \mathrm{~mm} \mathrm{Hg}$ ). On physical exam, he was conscious, with mild hypoactivity, no pallor, no cyanosis, clear ears but with erythematous pharynx, regular heart sounds with no audible murmur, good bilateral air entry with referred rhonchi, soft non-tender abdomen, non-distended, good muscle tone and no focal deficit with negative meningeal signs.

Lab tests taken upon admission showed $12,800 / \mathrm{mm}^{3}$ white blood count (WBC) with neutrophils of $49 \%$, lymphocytes of $32 \%$, platelets of $302,000 / \mathrm{mm}^{3}$, hemoglobin of $9.9 \mathrm{~g} / \mathrm{dL}$, erythrocyte sedimentation rate (ESR) of 90, C-reactive protein of 7.1 $\mathrm{mg} / \mathrm{dL}$, serum glutamate-pyruvate transaminase (SGPT) of 19 $\mathrm{U} / \mathrm{L}$, creatinine of $0.2 \mathrm{mg} / \mathrm{dL}$, Epstein-Barr virus IgM (EBV) of 0.6 , cytomegalovirus (CMV) of 0.19 and brucella titer: $<1: 20$; all were normal. Urine analysis and stool analysis were negative. Cultures taken from urine, stool and blood showed no growth.

After admission, fever continued. On day 9, the patient started to be hypoactive with edema of the right index and third finger and changed to be blue in color (Fig. 1). Labs where repeated showed an ESR of 100 and WBC of $18,000 / \mathrm{mm}^{3}$; urgent echocardiography was done, which showed left coronary artery dilatation of $2 \mathrm{~mm}$. 


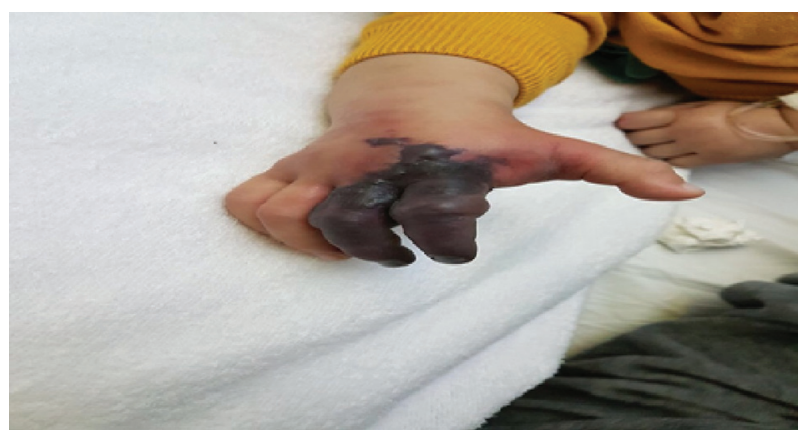

Figure 1. Dry gangrenous ischemia of the second and third fingers of right hand.

IVIG was given ( $2 \mathrm{~g} / \mathrm{kg})$, aspirin (100 mg/ $\mathrm{kg} /$ day $)$, prostaglandin E1 infusion $(0.02 \mathrm{mcg} / \mathrm{kg} / \mathrm{min})$, enoxaparin $(1 \mathrm{mg} / \mathrm{kg} /$ dose) every $12 \mathrm{~h}$, fresh frozen plasma once daily, broad spectrum antibiotics, meropenem (40 mg/ $/ \mathrm{kg} /$ dose) and vancomycin (20 mg/kg/dose) for 14 days, and pulse steroid therapy (30 $\mathrm{mg} / \mathrm{kg}$ ) given for 3 days.

On day 10, the patient had a significant increase in swelling associated with severe pain; thus, fasciotomy was done to relieve his compartment syndrome, and swab culture was taken which revealed no growth.

Coagulation profile showed normal international normalized ratio (INR), partial thromboplastin time (PTT), protein C, anti-thrombin III, fibrinogen, low protein $\mathrm{S}$ and D-dimer, and factor V Leiden heterozygous mutation.

Repeated echocardiography in 2 weeks was normal, and CT scan angiography of the right upper limb showed patent vessels (Fig. 2). The patient was discharged home on enoxa-

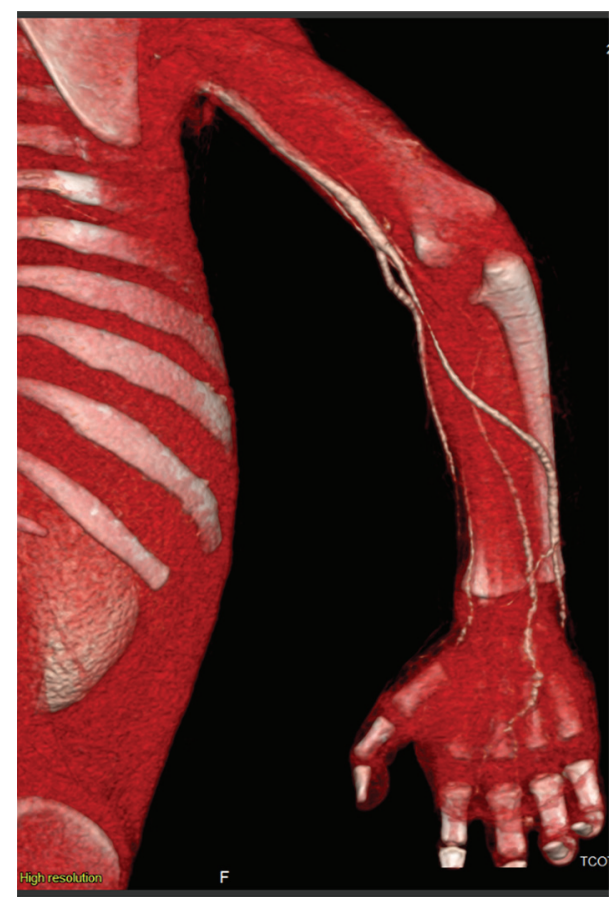

Figure 2. 3D reconstruction of $C T$ angiography revealing patent vessels of the right upper limb.

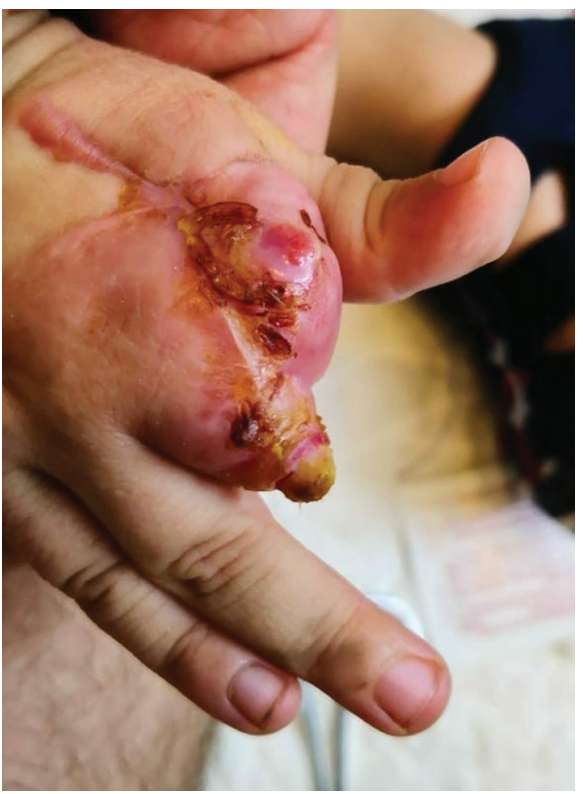

Figure 3. Autoamputation of the second and third right fingers after 5 months.

parin and aspirin, and was followed up by plastic surgeon; dry dressing was applied and the anti-factor Xa was monthly monitored to maintain a therapeutic range $(0.5-1.0 \mathrm{unit} / \mathrm{mL})$ of enoxaparin. Unfortunately, autoamputation of the second and third right fingers finally took place after 5 months (Fig. 3).

\section{Discussion}

Herein we report a case of patient with atypical Kawasaki, who developed peripheral ischemia of second and third fingers of right hand, a rare complication, and was found to have factor $\mathrm{V}$ Leiden heterozygous mutation that increases the risk of coagulopathy and thrombosis.

Early diagnosis and treatment of Kawasaki disease is important; however, an atypical form of Kawasaki disease is now well understood. To facilitate the diagnosis of incomplete or atypical Kawasaki disease, the algorithm recently published by the American Heart Association (AHA) [2, 3] calls for measurement of C-reactive protein and ESR on day 5 of fever in patients with 2 or 3 of the clinical criteria for Kawasaki disease. Patients found to have a C-reactive protein level of $3.0 \mathrm{mg} / \mathrm{dL}$ and/or an ESR of $40 \mathrm{~mm} / \mathrm{h}$ are advised to undergo supplemental laboratory testing and an echocardiogram. Patients meeting 3 or more of the supplementary laboratory criteria (albumin $3.0 \mathrm{~g} / \mathrm{dL}$, anemia for age, elevated alanine aminotransferase, platelets after 7 days $450,000 / \mathrm{mm}^{3}$, white blood cell count $15,000 / \mathrm{mm}^{3}$ and urine 10 white blood cells/high-power field) or with a positive echocardiogram should be treated with IVIG for Kawasaki disease [3].

This latter aspect of the algorithm is designed to "increase the sensitivity while maintaining sufficient specificity," given the potential serious complications of the disease and the safety and efficacy of early treatment [3]. We have identified 14 other case reports of peripheral gangrene in patients 
with Kawasaki disease. The median age of these patients with gangrene is 3 months [4]. Possible pathogenic mechanisms of peripheral gangrene include severe arteritis of digital or other peripheral small arteries; arteriospasm of peripheral smallto-medium-sized arteries, perhaps in association with severe vasculitis; thrombosis of inflamed or spastic small to mediumsized arteries as a result of stagnant blood flow and damaged endothelium; and thrombosis of a more proximal arterial aneurysm with embolism distally [5]. The median time was 15-31 days after the onset of illness for the clinical signs of peripheral ischemia and gangrene to manifest in the previously reported cases, despite initiating anti-inflammatory therapy [5].

There is no consensus regarding the ideal therapy for the peripheral ischemia in the aforementioned case reports [4]. In our case, it appeared that IVIG, high-dose aspirin, enoxaparin and pulse steroid therapy were most helpful in controlling the inflammatory process, and the fever resolved soon after initiation of these therapies.

The etiology of congenital atresia involving the jejunum, ileum and colon has long been thought to result from an in utero mesenteric vascular accident [6]. In 1912, Spuggs suggested that mechanical accidents, including vascular accidents, might be responsible for intestinal atresia [7]. An increased risk of thrombotic disease recently has been described in association with inherited thrombophilia, defined as a genetically inherited tendency towards spontaneous vascular thrombosis [8]. Before 1993, the chance of discovering a definable thrombophilia in patients with a thrombotic event was as low as $5-15 \%$ [6]. Thus, the chance of discovering an association between thrombophilia and a birth defect was extremely unlikely [9]. This has changed over the past few years with the discovery of numerous genetically transmissible factors that are known to predispose to thrombotic disease. Of these inherited thrombophilias, the best described and most common condition is referred to as factor $\mathrm{V}$ Leiden. The factor $\mathrm{V}$ Leiden mutation predisposes to thrombosis because of a loss of the anticoagulant properties of protein $\mathrm{C}$, which normally limit the coagulation cascade [10]. The pathophysiological mechanism by which activated protein $\mathrm{C}$ (APC) resistance occurs is related to an inherited abnormality of factor $\mathrm{V}$ rather than an intrinsic abnormality in APC. Among heterozygotes, thrombosis occurs at a rate of $5-10$ times that of the unaffected population (as our patient); with homozygotes, thrombosis occurs 50 - 100 times more frequently [11].

In conclusion, peripheral limb gangrene is a rare complication of Kawasaki disease, which needs to be early recognized and treated with every effective treatment including anti-inflammatory and anti-thrombotic drugs. We should search for inherited thrombophilias that might have an add-on role in the peripheral gangrene, and further aggressive treatment with plasma and factors replacement, to minimize the sequalae.

\section{Conflict of Interest}

The authors declare that they have no conflict of interest.

\section{Consent}

Written informed consent was obtained from the patient's family for publication of this case report and accompanying images.

\section{References}

1. Rowley AH, Gonzalez-Crussi F, Shulman ST. Kawasaki syndrome. Rev Infect Dis. 1988;10(1):1-15.

2. Newburger JW, Takahashi M, Gerber MA, Gewitz MH, Tani LY, Burns JC, Shulman ST, et al. Diagnosis, treatment, and long-term management of Kawasaki disease: a statement for health professionals from the Committee on Rheumatic Fever, Endocarditis, and Kawasaki Disease, Council on Cardiovascular Disease in the Young, American Heart Association. Pediatrics. 2004;114(6):17081733.

3. Newburger JW, Takahashi M, Gerber MA, Gewitz MH, Tani LY, Burns JC, Shulman ST, et al. Diagnosis, treatment, and long-term management of Kawasaki disease: a statement for health professionals from the Committee on Rheumatic Fever, Endocarditis and Kawasaki Disease, Council on Cardiovascular Disease in the Young, American Heart Association. Circulation. 2004;110(17):27472771.

4. Durall AL, Phillips JR, Weisse ME, Mullett CJ. Infantile Kawasaki disease and peripheral gangrene. J Pediatr. 2006;149(1):131-133.

5. Tomita S, Chung K, Mas M, Gidding S, Shulman ST. Peripheral gangrene associated with Kawasaki disease. Clin Infect Dis. 1992;14(1):121-126.

6. Johnson SM, Meyers RL. Inherited thrombophilia: a possible cause of in utero vascular thrombosis in children with intestinal atresia. J Pediatr Surg. 2001;36(8):11461149.

7. Abrams JS. Experimental intestinal atresia. Surgery. 1968;64(1):185-191.

8. Kodish E, Potter C, Kirschbaum NE, Foster PA. Activated protein $\mathrm{C}$ resistance in a neonate with venous thrombosis. J Pediatr. 1995;127(4):645-648.

9. Koster T, Rosendaal FR, de Ronde H, Briet E, Vandenbroucke JP, Bertina RM. Venous thrombosis due to poor anticoagulant response to activated protein $\mathrm{C}$ : Leiden Thrombophilia Study. Lancet. 1993;342(88868887):1503-1506.

10. Bick RL, Kaplan H. Syndromes of thrombosis and hypercoagulability. Congenital and acquired causes of thrombosis. Med Clin North Am. 1998;82(3):409-458.

11. Ridker PM, Miletich JP, Stampfer MJ, Goldhaber SZ, Lindpaintner K, Hennekens CH. Factor V Leiden and risks of recurrent idiopathic venous thromboembolism. Circulation. 1995;92(10):2800-2802. 